\title{
QUALIDADE DE MAÇÃS 'FUJI' INFLUENCIADA PELA ADUBAÇÃO NITROGENADA E POTÁSSICA EM DOIS TIPOS DE SOLO ${ }^{1}$
}

\author{
FRANCIELLE DE SOUZA², LUIZ CARLOS ARGENTA ${ }^{3}$, GILBERTO NAVA ${ }^{4}$, \\ PAULO ROBERTO ERNANI ${ }^{5}$, CASSANDRO VIDAL TALAMINI DO AMARANTE ${ }^{5}$
}

RESUMO - Macieiras 'Fuji' crescidas sobre Cambissolo e Neossolo foram tratadas com doses de N e $\mathrm{K}_{2} \mathrm{O}\left(0 ; 50 ; 100\right.$ e $200 \mathrm{~kg} \mathrm{ha}^{-1}$ de ambos) por nove anos. As alterações da maturação e qualidade das maçãs, na colheita e após a armazenagem, decorrentes das aplicações de $\mathrm{N}$ e $\mathrm{K}_{2} \mathrm{O}$, não foram iguais para os dois solos. Altas doses de $\mathrm{N}$ aumentaram o teor de $\mathrm{N}$ e diminuíram a cor vermelha e o teor de amido nos frutos, independentemente do solo e da dose de $\mathrm{K}_{2} \mathrm{O}$. A massa dos frutos aumentou, e a firmeza da polpa diminuiu em resposta às altas doses de $\mathrm{N}$ aplicadas no Neossolo, independentemente da dose de $\mathrm{K}_{2} \mathrm{O}$, o que não ocorreu de forma consistente no Cambissolo. A acidez titulável (AT) reduziu por altas doses de $\mathrm{N}$ em ambos os solos, quando se aplicaram baixas doses de $\mathrm{K}_{2} \mathrm{O}$. Os teores de $\mathrm{K}$, amido, AT, massa e coloração avermelhada aumentaram, enquanto a firmeza diminuiu consistentemente e independentemente da dose de $\mathrm{N}$, em resposta a altas doses de $\mathrm{K}_{2} \mathrm{O}$ no Cambissolo, mas não no Neossolo. Os efeitos de doses de $\mathrm{N}_{\text {e }} \mathrm{K}_{2} \mathrm{O}$ sobre a firmeza da polpa e AT foram mais evidentes após a armazenagem do que na colheita.

Termos de indexação: Malus domestica, nutrição, nitrogênio, potássio, maturação de fruto, armazenagem.

\section{QUALITY OF 'FUJI' APPLES AFFECTED BY NITROGEN AND POTASSIUM FERTILIZATION IN TWO SOIL TYPES}

\begin{abstract}
Fuji' apple trees grown on Inceptisol and Entisol were annually fertilized with doses of N and $\mathrm{K}_{2} \mathrm{O}\left(0,50,100\right.$, and $200 \mathrm{~kg} \mathrm{ha}^{-1}$, for both) along nine growing seasons. Changes of fruit maturity and quality at harvest and after cold storage in response to $\mathrm{N}$ and $\mathrm{K}_{2} \mathrm{O}$ fertilization were not the same for the two soil types. The increment of $\mathrm{N}$ rate applied increased the $\mathrm{N}$ content and reduced the starch content and peel red color of the fruit in both soils, regardless of $\mathrm{K}_{2} \mathrm{O}$ rate. On the Entisol, the increment of $\mathrm{N}$ rate increased the fresh mass and reduced the flesh firmness of the fruit, regardless of $\mathrm{K}_{2} \mathrm{O}$ rate, while in the Inceptisol this response was not consistent. Fruit titratable acidity (TA) reduced with the increment of $\mathrm{N}$ rate in both soils, especially at low rates of $\mathrm{K}_{2} \mathrm{O}$. For the orchard on Inceptisol, the $\mathrm{K}$ and starch contents, TA, fresh mass, and peel red color increased, while the flesh firmness reduced consistently and independently of $\mathrm{N}$ rate, with the increment of $\mathrm{K}_{2} \mathrm{O}$ rate, but not for the orchard on the Entisol. The effects of $\mathrm{N}$ and $\mathrm{K}_{2} \mathrm{O}$ rates on flesh firmness and TA of the fruit were more evident after cold storage than at harvest.
\end{abstract}

Index terms: Malus domestica, nutrition, nitrogen, potassium, fruit maturity, storage.

\footnotetext{
${ }^{1}$ (Trabalho 214-12). Recebido para publicação em: 05-07-2012. Aceito para publicação em: 31-08-2012.

${ }^{2}$ Aluna do Curso de Mestrado em Fisiologia Vegetal, Departamento de Botânica, Universidade Federal de Pelotas (UFPel), Cx. Postal 354, CEP 96010-900, Pelotas, RS. E-mail:frandelotus@ibest.com.br

${ }^{3}$ Dr., Pesquisador da Empresa de Pesquisa Agropecuária e Extensão Rural e Santa Catarina S.A. (EPAGRI), Estação Experimental de Caçador, Cx. Postal 591, CEP 89500-000, Caçador, SC. E-mail: argenta@epagri.sc.gov.br

${ }^{4}$ Dr., Pesquisador EPAGRI, Estação Experimental de São Joaquim, Cx. Postal 81, CEP 88600-000, São Joaquim, SC. E-mail: nava@ epagri.sc.gov.br

${ }^{5}$ Ph.D., Bolsista de Produtividade em Pesquisa do CNPq, Professor da Universidade do Estado de Santa Catarina (UDESC). Av. Luiz de Camões, 2090, CEP 88520-000, Lages, SC. E-mails: a2pre@cav.udesc.br; amarante@cav.udesc.br
} 


\section{INTRODUÇÃO}

Na região serrana de Santa Catarina, ocorrem as condições climáticas mais favoráveis à produção de maçãs do Brasil. No entanto, grande parte dos solos dessa região são rasos, pedregosos e deficientes para alguns nutrientes minerais em relação aos solos mais profundos e com menos pedras nas camadas ocupadas pelas raízes. Quando o solo não supre quantitativamente a alta demanda das macieiras por minerais, tais como nitrogênio $(\mathrm{N})$ e potássio $(\mathrm{K})$, eles têm de ser suplementados pela adição de fertilizantes (ERNANI et al., 2002; NAVA et al., 2008).

A qualidade de maçãs por ocasião da colheita é influenciada por fatores inerentes à cultivar, ao clima, às técnicas de manejo das plantas, ao estádio de maturação (WARGO et al., 2003) e à disponibilidade de nutrientes do solo (ERNANI et al., 2002; FERGUSON; BOYD, 2002). A conservação da qualidade após a colheita é afetada pelos mesmos fatores relacionados e pelas condições de armazenagem, incluindo a temperatura e a concentração dos gases nas câmaras frigoríficas sob condição de atmosfera controlada (AC) (LITTLE; HOLMES, 2000).

Em solos profundos da região Sul do Brasil, a adição de $\mathrm{N}$ não tem aumentado o rendimento de maçãs (BASSO; SUZUKI, 1992; ERNANI; DIAS, 1999; ERNANI et al., 2000), diferentemente do que ocorre em solos pouco profundos e pedregosos (NAVA et al., 2008; NAVA; DECHEN, 2009). Alta disponibilidade de $\mathrm{N}$ nos solos pode afetar tanto a produtividade quanto a qualidade dos frutos por influenciar positivamente o tamanho (JOHNSON, 1996; WARGO et al., 2003), negativamente a cor vermelha e a firmeza da polpa (JOHNSON, 1996; DRAKE et al., 2002; WARGO et al., 2003), e por favorecer o desenvolvimento de distúrbios fisiológicos (RAESE; DRAKE, 1997). O excesso de N também pode diminuir a longevidade das maçãs em câmaras frigoríficas, por favorecer a perda da firmeza da polpa, o desenvolvimento de distúrbios fisiológicos e doenças, a respiração e a produção de etileno (LITTLE; HOLMES, 2000, FALLAHI et al., 2010).

A disponibilidade de $\mathrm{K}$ às plantas em solos altamente intemperizados, com predomínio de cargas variáveis, depende basicamente da concentração do nutriente no solo, do volume de solo ocupado pelas raízes e do teor de água volumétrica (ERNANI et al., 2002). Como a reserva de K nos solos brasileiros é pequena e esgota- se em poucos anos, ele tem de ser adicionado por meio de fertilizantes a fim de manter altas produtividades de frutos (ERNANI et al., 2002). A deficiência de $\mathrm{K}$, além de limitar a produtividade (ERNANI et al., 2002; NAVA; DECHEN, 2009), pode prejudicar o tamanho e a coloração das maçãs (HUNSCHE et al., 2003), enquanto seu excesso pode favorecer o aparecimento de distúrbios fisiológicos e limitar a longevidade dos frutos, por diminuir a firmeza da polpa (HUNSCHE et al., 2003). Os efeitos negativos associados aos excessos de adubação com $\mathrm{N}$ e K, na qualidade das maçãs, nem sempre é verificado, principalmente nas condições brasileiras (ERNANI et al., 2002).

O objetivo deste trabalho foi avaliar os efeitos interativos de doses de $\mathrm{N}$ e $\mathrm{K}_{2} \mathrm{O}$, em dois tipos de solos da região serrana de Santa Catarina, sobre a qualidade de maçãs 'Fuji' na colheita e após a armazenagem sob atmosfera do ar (AA) e atmosfera controlada (AC).

\section{MATERIAL E MÉTODOS}

$\mathrm{O}$ estudo foi conduzido em dois pomares de macieiras da cultivar 'Fuji', sobre porta-enxerto 'Marubakaido', localizados na região de São Joaquim-SC (28 $17^{\prime} 25^{\prime}$ ' S, 49 $9^{\circ} 56^{\prime} 56^{\prime}$ ” W), numa altitude aproximada de $1.350 \mathrm{~m}$. Um dos pomares foi plantado em 1992, sobre um Cambissolo Húmico, e o outro em 1996, sobre um Neossolo. Os dois solos eram pouco profundos e possuíam pedras soltas na camada superficial. O sistema de manejo recomendado pelos órgãos oficiais de extensão rural para pomares comerciais foi igualmente adotado para ambos os pomares, exceto em relação às adubações. Previamente ao plantio das árvores, foi aplicado calcário dolomítico e fertilizantes minerais, conforme recomendações técnicas para a cultura da macieira (CQFS, 2004), para aumentar o $\mathrm{pH}$ do solo, eliminar a toxidez de $\mathrm{Al}$ e $\mathrm{Mn}$, e elevar a disponibilidade de $\mathrm{P}$ e K. As características físico-químicas dos dois solos, relativas ao tratamento-testemunha (sem adição de $\mathrm{N}$ e $\mathrm{K}_{2} \mathrm{O}$ ), são descritas na Tabela 1.

Os tratamentos consistiram em uma combinação fatorial de doses de $\mathrm{N} \mathrm{e} \mathrm{K}_{2} \mathrm{O}$ : 0; 50; 100 e 200 $\mathrm{kg} \mathrm{ha}^{-1}$, para ambos. Os nutrientes foram aplicados anualmente, de 1998 a 2006, sem incorporação com o solo, numa faixa de 2,2 m de largura, centralizada junto à linha de plantas, sobre a área correspondente à projeção da copa das árvores. $\mathrm{O} \mathrm{K}_{2} \mathrm{O}$ foi sempre aplicado após a colheita dos frutos, em abril, na forma de cloreto de potássio. $\mathrm{O} \mathrm{N}$ foi parcelado igualitariamente em duas vezes, tendo sido aplicado após a colheita (abril) e no início da estação de crescimento (setembro), na forma de ureia. Nos dois pomares, as unidades experimentais foram constituídas por cinco plantas, tendo sido colhidos apenas frutos das três plantas centrais. Todas as parcelas receberam $50 \mathrm{~kg} \mathrm{ha}^{-1} \mathrm{de}_{2} \mathrm{O}_{5}$, na forma de superfosfato triplo, 
juntamente com a aplicação de $\mathrm{K}_{2} \mathrm{O}$.

Em 2005 e 2006, os frutos de todos os tratamentos foram colhidos num mesmo dia. O ponto de colheita foi definido com base no índice de amido dos frutos do tratamento- testemunha (que não recebeu aplicações de $\mathrm{Ne} \mathrm{K}_{2} \mathrm{O}$ ) do pomar sobre Neossolo. $\mathrm{O}$ índice de amido foi determinado por meio da comparação do escurecimento da metade peduncular dos frutos, tratada com uma solução de iodo, em uma escala de 1 (teor máximo de amido) a 9 (amido totalmente hidrolisado). Os frutos foram colhidos quando este índice atingiu valores entre 3,5 e 6,0, e armazenados a $0,5{ }^{\circ} \mathrm{C} / 90-95 \%$ UR sob AA por 195 dias, e sob AC $\left(1,5 \mathrm{kPa} \mathrm{O}_{2}+2,0 \mathrm{kPa} \mathrm{CO}\right)$ por 225 dias, em câmaras comerciais.

A qualidade e a maturação dos frutos foram avaliadas no dia seguinte à colheita e após a armazenagem refrigerada sob AA e AC, mais sete dias sob AA a $23^{\circ} \mathrm{C}$. A massa fresca, o índice de amido (apenas na colheita), a firmeza da polpa, os teores de sólidos solúveis (SS) e a acidez titulável (AT) foram quantificados conforme descrito por Argenta et al. (2010). A proporção de cor vermelha da película foi estimada visualmente apenas na colheita, sendo expressa em percentagem de área avermelhada relativa à superfície total do fruto. Os teores de $\mathrm{Ca}, \mathrm{K}, \mathrm{Mg}$, $\mathrm{Ne} \mathrm{P}$ ( $\mathrm{mg} \mathrm{kg}^{-1}$ de massa fresca) foram determinados em uma fatia longitudinal de $1 \mathrm{~cm}$ de espessura, em forma de cunha, com casca, sem a parte central do carpelo, extraída de cada fruto. A digestão da amostra $(\sim 5,0 \mathrm{~g})$ foi feita utilizando-se de $\mathrm{H}_{2} \mathrm{SO}_{4}+$ $\mathrm{H}_{2} \mathrm{O}_{2}$, segundo a metodologia descrita por Tedesco et al. (1995). Os teores de $\mathrm{N}$ foram determinados por semimicro-Kjeldahl; os teores de $\mathrm{K}, \mathrm{Mg}$ e $\mathrm{Ca}$, por espectrofotometria de absorção atômica; e os teores de P pelo método do vanadato-molibdato de amônio.

Cada repetição foi composta de 120 frutos para as análises da massa, 40 frutos para as análises da cor, firmeza e amido, e três subamostras de 10 frutos para análises dos teores de SS, AT e minerais. Foi utilizado o delineamento experimental em blocos ao caso, com cinco repetições, em esquema fatorial 4 $\mathrm{x} 4$, correspondendo às doses de $\mathrm{N} \mathrm{e}_{2} \mathrm{O}$. Os dados de 2005 e 2006, de cada tipo de solo (Cambissolo e Neossolo), foram submetidos à análise de variância (SAS Institute, Inc.). Os efeitos de doses de $\mathrm{Ne} \mathrm{K}_{2} \mathrm{O}$ foram analisados por contrastes ortogonais.

\section{RESULTADOS E DISCUSSÃO}

Os dados apresentaram comportamento similar nos anos de 2005 e 2006 , e não houve interação entre tratamentos (doses de $\mathrm{N} \mathrm{e}_{2} \mathrm{O}$ ) e anos para os atributos de composição mineral, maturação e qualidade dos frutos, em ambos os pomares. Por isso, a análise estatística foi feita utilizando a combinação dos dois anos, e avaliando apenas o efeito de doses de $\mathrm{N}$ e $\mathrm{K}_{2} \mathrm{O}$ em cada pomar.

A composição química dos frutos de plantastestemunha (que não receberam aplicações de $\mathrm{N}$ e $\mathrm{K}_{2} \mathrm{O}$ ) diferiu entre os dois pomares. Frutos de plantas-testemunhas crescidas sobre Cambissolo apresentaram menores teores de $\mathrm{K}$ e de $\mathrm{P}$, maior teor de $\mathrm{N}$ e teores similares de $\mathrm{Ca}$ e $\mathrm{Mg}$ em relação àqueles crescidos sobre Neossolo (Tabela 2). A diferença entre frutos-testemunha crescidos sobre Cambissolo e Neossolo quanto à composição de $\mathrm{K}$ e P relacionaram- se com as variações do conteúdo desses nutrientes nos respectivos solos (Tabela 1). No entanto, as diferenças entre os dois solos quanto ao conteúdo desses dois nutrientes foram muito maiores que as respectivas variações nos frutos. As concentrações de P e K disponíveis no Neossolo foram aproximadamente 2 e 4,9 vezes maiores que aquelas no Cambissolo (Tabela 1). Já os teores de $\mathrm{P}$ e K nos frutos crescidos sobre Neossolo foram apenas $6 \%$ e 1,7 vezes maiores que aquelas nos frutos crescidos sobre Cambissolo (Tabela 2).

$\mathrm{O}$ teor de $\mathrm{N}$ nos frutos aumentou com a adição desse nutriente, nos dois solos e para todas as doses de $\mathrm{K}_{2} \mathrm{O}$, porém com diferentes magnitudes (Tabela 2). A adição de cada $10 \mathrm{~kg} \mathrm{ha}^{-1}$ de $\mathrm{N}$ no Cambissolo promoveu incrementos de 7,5 e 2,0 $\mathrm{mg} \mathrm{kg}^{-1}$ de $\mathrm{N}$ nos frutos, quando as doses de $\mathrm{K}_{2} \mathrm{O}$ aplicadas foram 0 e $200 \mathrm{~kg} \mathrm{ha}^{-1}$, respectivamente. Já a adição de cada $10 \mathrm{~kg} \mathrm{ha}^{-1}$ de $\mathrm{N}$ no Neossolo promoveu incrementos de 4,5 e 2,8 $\mathrm{mg} \mathrm{kg}^{-1}$ de $\mathrm{N}$ nos frutos, quando as doses de $\mathrm{K}_{2} \mathrm{O}$ aplicadas foram 0 e $200 \mathrm{~kg} \mathrm{ha}^{-1}$, respectivamente. No pomar sobre o Cambissolo, o incremento das doses de $\mathrm{N}$ diminuiu os teores de $\mathrm{P}$ e $\mathrm{K}$, e não alterou consistentemente os teores de $\mathrm{Ca}$ e Mg nos frutos.

$\mathrm{O}$ teor de $\mathrm{K}$ nos frutos não foi alterado em resposta à adição de $\mathrm{K}_{2} \mathrm{O}$ no Neossolo, mas aumentou linearmente em resposta à adição de $\mathrm{K}_{2} \mathrm{O}$ no Cambissolo, independentemente da dose de $\mathrm{N}$ (Tabela 2). Essas diferenças de respostas à adição de $\mathrm{K}_{2} \mathrm{O}$ foi relacionada às diferenças entre os solos quanto à disponibilidade de $\mathrm{K}$ (Tabela 1 ). No Cambissolo, a adição de cada $10 \mathrm{~kg} \mathrm{ha}^{-1}$ de $\mathrm{K}_{2} \mathrm{O}$ resultou em aumento de 24,7 e $37,2 \mathrm{mg} \mathrm{kg}^{-1} \mathrm{de} \mathrm{K}^{2}$ nos frutos, quando as doses de $\mathrm{N}$ aplicadas foram 0 e $200 \mathrm{~kg} \mathrm{ha}^{-1}$, respectivamente. A aplicação de doses crescentes de $\mathrm{K}_{2} \mathrm{O}$ não afetou os teores dos demais nutrientes analisados na maioria das doses de N, no pomar sobre Neossolo, mas reduziu o teor de $\mathrm{Ca}$, dependendo da dose de $\mathrm{N}$, no pomar sobre Cambissolo (Tabela 2). 
A alteração do tamanho dos frutos em resposta à adição de $\mathrm{Ne} \mathrm{K}_{2} \mathrm{O}$ diferiu entre os pomares (Tabela $3)$, com interação significativa entre doses de $\mathrm{Ne}$ $\mathrm{K}_{2} \mathrm{O}(\mathrm{p}<0,001)$. O aumento da dose de $\mathrm{N}$ aumentou a massa dos frutos crescidos sobre Neossolo, independentemente da dose de $\mathrm{K}_{2} \mathrm{O}$. No pomar sobre Cambissolo, entretanto, o incremento na dose de $\mathrm{N}$ não afetou a massa dos frutos, quando as doses de $\mathrm{K}_{2} \mathrm{O}$ foram 100 e $200 \mathrm{~kg} \mathrm{ha}^{-1}$, e diminuiu a massa dos frutos, quando as doses de $\mathrm{K}_{2} \mathrm{O}$ foram 0 e $50 \mathrm{~kg} \mathrm{ha}^{-1}$. Por outro lado, a massa dos frutos foi aumentada quando crescidos sobre Cambissolo e não alterada quando crescidos sobre Neossolo, em resposta ao aumento da dose de $\mathrm{K}_{2} \mathrm{O}$, independentemente da dose de $\mathrm{N}$. A variação dos efeitos da adição de $\mathrm{K}_{2} \mathrm{O}$ entre os pomares sobre a massa dos frutos foi associada à disponibilidade de K no solo. Ou seja, houve aumento da massa dos frutos em resposta ao aumento da dose de $\mathrm{K}_{2} \mathrm{O}$ apenas para o solo com menor teor de $\mathrm{K}$ disponível (Cambissolo). A variação dos efeitos da adição de $\mathrm{N}$ entre os pomares não foi relacionada aos teores de matéria orgânica dos solos, indicando que este não é um parâmetro preciso, quando utilizado isoladamente, para predizer a disponibilidade de $\mathrm{N}$.

$\mathrm{O}$ aumento da massa dos frutos em resposta à adição de $\mathrm{N}$ observado no pomar sobre Neossolo é semelhante àquela observada na América do Norte (JOHNSON, 1996; RAESE et al., 2007). Ausência de resposta à adição de doses de $\mathrm{N}$ sobre o crescimento de maçãs, semelhante àquelas observadas no pomar sobre Cambissolo (Tabela 3), também foi descrita para pomares sobre Latossolos das regiões de Fraiburgo-SC (BASSO; SUZUKI, 1992) e Vacaria-RS (ERNANI; DIAS, 1999). A redução do tamanho dos frutos pelo aumento da dose de $\mathrm{N}$ no pomar sobre Cambissolo, quando as doses de $\mathrm{K}_{2} \mathrm{O}$ foram 0 e $50 \mathrm{~kg} \mathrm{ha}^{-1}$, pode estar relacionada à deficiência de $\mathrm{K}$ (elemento limitante) nesse solo. É possível que sob severa deficiência de $\mathrm{K}$, a adição de $\mathrm{N}$ favoreça o crescimento vegetativo em detrimento do crescimento dos frutos. Redução do tamanho de maçãs 'Delicious' em resposta a doses excessivamente altas de $\mathrm{N}$ foi observada por Fallahi (2000). A competição por fotoassimilados entre brotações e frutos pode ser estimulada em resposta à adubação com $\mathrm{N}$, quando feita na fase de maior crescimento vegetativo (FALLAHI et al., 2010).

$\mathrm{O}$ efeito da adubação com $\mathrm{N}$ e $\mathrm{K}_{2} \mathrm{O}$ sobre o índice de iodo-amido dos frutos na colheita variou com o tipo de solo (Tabela 3). O aumento da dose de $\mathrm{K}_{2} \mathrm{O}$ diminuiu o índice de iodo-amido (aumentou o teor de amido), independentemente da dose de $\mathrm{N}$, no pomar sobre Cambissolo, e em duas das quatro doses de $\mathrm{N}$ no pomar sobre Neossolo (Tabela 3). O efeito do N, entretanto, foi mais evidente do que o do $\mathrm{K}_{2} \mathrm{O}$, tendo havido aumento do índice de iodoamido (redução do teor de amido) com o aumento da dose de $\mathrm{N}$, independentemente da dose de $\mathrm{K}_{2} \mathrm{O}$, nos dois pomares, com uma exceção da dose de 100 $\mathrm{kg} \mathrm{ha}^{-1}$ de $\mathrm{K}_{2} \mathrm{O}$ no Neossolo, onde não houve efeito da adição de $\mathrm{N}$. O aumento do índice de iodo-amido em resposta a altas doses de $\mathrm{N}$, observado em várias cultivares de maçãs, incluindo 'Fuji' (RAESE; DRAKE, 1997) e 'Gala' (NEILSEN et al., 2000), está associado ao aumento da produção de etileno (FALLAHI, 2000). Isso sustenta a hipótese de que altas doses de $\mathrm{N}$ aceleram a maturação e a degradação do amido (LITTLE; HOLMES, 2000). Da mesma forma, o menor índice de iodo-amido pode indicar retardamento da maturação, associada ao aumento da dose de $\mathrm{K}_{2} \mathrm{O}$. Entretanto, é importante considerar que os efeitos do $\mathrm{N}$ e do $\mathrm{K}_{2} \mathrm{O}$ sobre o teor de amido no fruto podem não estar relacionados apenas à alteração da taxa de sua degradação durante a maturação, mas também ao seu acúmulo durante o período de crescimento dos frutos. Aparentemente, é possível que altas doses de $\mathrm{K}$ favoreçam o acúmulo de açúcares solúveis e amido nos frutos, pelo estímulo do transporte de açúcares a partir das folhas e/ou pelo aumento da fotossíntese (FAUST, 1989; TAIZ; ZEIGER, 2006). Por outro lado, o estímulo no crescimento vegetativo, proporcionado por altas doses de N, pode favorecer o transporte de açúcares para as gemas vegetativas, resultando no menor acúmulo de amido nos frutos (FAUST, 1989).

O desenvolvimento da cor vermelha na superfície dos frutos foi reduzida em resposta ao aumento da dose de $\mathrm{N}$, independentemente do pomar e da dose de $\mathrm{K}_{2} \mathrm{O}$ (Tabela 3). $\mathrm{O}$ efeito do aumento da dose de $\mathrm{K}_{2} \mathrm{O}$ na coloração, entretanto, variou com o tipo de solo e com a dose de N. No pomar sobre Cambissolo, a cor vermelha aumentou em resposta ao aumento da dose de $\mathrm{K}_{2} \mathrm{O}$, independentemente da dose de $\mathrm{N}$ aplicada, e no pomar sobre Neossolo, aumentou linearmente com a dose de $\mathrm{K}_{2} \mathrm{O}$ apenas quando foram aplicados 0 e $100 \mathrm{~kg}$ de $\mathrm{N}$.

A menor cor vermelha nos frutos de pomares que recebem alta dose de $\mathrm{N}$ pode estar relacionada ao maior crescimento vegetativo, proporcionado por este nutriente, que reduz a incidência de radiação no interior das plantas e, assim, o acúmulo de antocianinas nos frutos (JOHNSON, 1996; FALLAHI, 2000). Adicionalmente, altos teores de $\mathrm{N}$ favorecem o acúmulo de clorofila e reduzem a revelação de antocianinas nos frutos (FERGUSON; BOYD, 2002).

O aumento da coloração avermelhada causado pela adubação potássica em solos deficientes 
nesse nutriente tem sido observado em vários estudos, embora nem sempre de forma consistente (NEILSEN et al., 1998; HUNSCHE et al., 2003; NEILSEN; NEILSEN, 2006; NAVA et al., 2008). O maior acúmulo de açúcares nos frutos, em resposta à adubação potássica, pode promover a síntese de antocianinas (FAUST, 1989), já que uma das características estruturais das antocianinas é possuir açúcar na posição 3 (TAIZ; ZEIGER, 2006).

Houve interação entre doses de $\mathrm{N}_{\text {e }} \mathrm{K}_{2} \mathrm{O}$ sobre a firmeza da polpa dos frutos $(\mathrm{p}<0,05)$. O efeito da

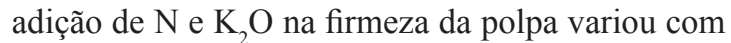
o pomar e com a época em que os frutos foram avaliados (Tabela 4). No pomar sobre Neossolo, a firmeza da polpa foi reduzida com o aumento da dose de $\mathrm{N}$, independentemente da dose de $\mathrm{K}_{2} \mathrm{O}$ e da época de análise dos frutos (Tabela 4). Exceção ocorreu para frutos do tratamento com $200 \mathrm{~kg} \mathrm{ha}^{-1} \mathrm{de}_{2} \mathrm{O}$, nos quais a redução da firmeza devido ao aumento da dose de $\mathrm{N}$ foi observada apenas após a armazenagem. No pomar sobre Cambissolo, os efeitos da adição de $\mathrm{N}$ sobre a firmeza da polpa não foram consistentes. Observou-se pequeno aumento da firmeza da polpa com o aumento da dose de $\mathrm{N}$, quando as doses de $\mathrm{K}_{2} \mathrm{O}$ foram 0 e $100 \mathrm{~kg} \mathrm{ha}^{-1}$, na colheita, e pequena redução da firmeza da polpa com o aumento da dose de $\mathrm{N}$, independentemente da dose de $\mathrm{K}_{2} \mathrm{O}$, após a armazenagem sob AA (Tabela 4).

A firmeza da polpa foi reduzida pelo aumento da dose de $\mathrm{K}_{2} \mathrm{O}$, independentemente da dose de $\mathrm{N}$ e da época de análise dos frutos crescidos sobre o Cambissolo, mas, normalmente, não foi alterada pelo aumento da dose de $\mathrm{K}_{2} \mathrm{O}$ nos frutos crescidos sobre o Neossolo (Tabela 4). As reduções da firmeza da polpa em resposta ao aumento da dose de $\mathrm{N}$ no pomar sobre Neossolo e em resposta ao aumento da dose de $\mathrm{K}_{2} \mathrm{O}$ no pomar sobre Cambissolo, estão relacionadas ao aumento do tamanho dos frutos. A concentração de materiais de parede celular e a firmeza da polpa, podem diminuir com o aumento do tamanho dos frutos, devido ao alongamento celular excessivo (TAIZ; ZEIGER, 2006). Em ambos os casos, os efeitos negativos de altas doses de $\mathrm{N}$ e de altas doses de $\mathrm{K}_{2} \mathrm{O}$ sobre a firmeza da polpa foram mais evidentes após a armazenagem, especialmente sobre AA, indicando que altas doses de $\mathrm{N} \mathrm{e}_{2} \mathrm{O}$ podem aumentar as taxas de perda de firmeza durante a armazenagem. Da mesma forma, o pequeno aumento da firmeza em resposta ao aumento da dose de $\mathrm{N}$, observado quando não se aplicou $\mathrm{K}_{2} \mathrm{O}$ no pomar sobre Cambissolo, foi relacionadas à redução da massa dos frutos. A variação da expressão dos efeitos de altas doses de $\mathrm{N}$ sobre a firmeza dos frutos armazenados sob AA, em relação àqueles analisados na colheita, quando crescidos sobre o Cambissolo, novamente indica que altas doses de $\mathrm{N}$ aumentam a perda pós-colheita de firmeza.

Ressalta-se que a redução da firmeza da polpa dos frutos crescidos sobre Cambissolo, em resposta ao aumento de doses de $\mathrm{K}_{2} \mathrm{O}$ (Tabela 4), pode também estar associada à redução do teor de Ca nos frutos (Tabela 2). Altos teores de Ca na polpa conferem maior firmeza ao fruto na colheita e menor perda de firmeza durante a armazenagem, pois o $\mathrm{Ca}$ é constituinte da parede celular (FERGUSON; BOYD, 2002).

A diminuição da firmeza de polpa pelo aumento da adubação nitrogenada também tem sido observada para outras cultivares de maçãs, embora a magnitude de seus efeitos varie com o ano, clima e época de aplicação do adubo (RAESE; DRAKE, 1997; FALLAHI, 2000; NEILSEN et al., 2000; RAESE et al., 2007). Algumas vezes, entretanto, não tem sido observada alteração da firmeza da polpa, nem tampouco do crescimento dos frutos, pelo aumento da adubação nitrogenada (FALLAHI et al., 2001). Em relação ao K, os resultados de estudos anteriores também são variáveis: em algumas situações, altas doses de K diminuem a firmeza da polpa de maçãs (HUNSCHE et al., 2003; NEILSEN; NEILSEN, 2006), enquanto noutras este efeito não ocorre (NEILSEN et al., 1998). A ausência de efeitos da adubação nitrogenada e potássica sobre o crescimento e a firmeza da polpa de maçãs observada em alguns estudos (BASSO; SUZUKI, 1992; ERNANI; DIAS, 1999; NEILSEN et al., 1998; FALLAHI et al., 2001) pode estar relacionada à maior disponibilidade desses nutrientes nos solos e/ou a ocorrência de outros fatores climáticos limitantes ao desenvolvimento dos frutos.

A AT aumentou com o incremento na dose de $\mathrm{K}_{2} \mathrm{O}$ aplicada no pomar sobre Cambissolo, independentemente da dose de $\mathrm{N}$ e época de análise dos frutos (Tabela 5). Esse efeito do $\mathrm{K}_{2} \mathrm{O}$ foi significativo no pomar sobre Neossolo apenas quando se aplicaram altas doses de $\mathrm{N}$, especialmente depois da armazenagem sob AC. Correlação positiva entre fertilização potássica e AT dos frutos tem sido observada em pomares de macieira 'Summerland McIntosh' (NEILSEN et al., 1998), 'Gala', 'Fiesta', 'Spartan' (NEILSEN; NEILSEN, 2006) e 'Fuji' (HUNCHE et al., 2003; NAVA et al., 2008). O aumento da concentração de $\mathrm{K}^{+}$no citosol e vacúolo em resposta à adubação potássica pode induzir a produção de ácidos orgânicos para a manutenção do equilíbrio entre cátions e ânions (FAUST, 1989).

A AT foi reduzida pela aplicação de doses crescentes de $\mathrm{N}$, dependendo do pomar e da dose de 
$\mathrm{K}_{2} \mathrm{O}$ (Tabela 5). Redução da AT, associada a aumento da adubação com $\mathrm{N}$, foi mais frequente quando se aplicaram baixas doses de $\mathrm{K}_{2} \mathrm{O}$. Da mesma forma, estudos anteriores indicam que aplicação de altas doses de $\mathrm{N}$ pode causar redução da $\mathrm{AT}$ em maçãs 'Gala' (NEILSEN et al., 2000) e 'Golden Delicious' (RAESE et al., 2007). Embora esse efeito tenha variado entre anos, a redução da AT pode ter resultado da diluição causada pelo aumento do tamanho dos frutos.

O teor de sólidos solúveis (SS) nos frutos foi o atributo menos influenciado, especialmente pela adubação nitrogenada (Tabela 6). O teor de SS aumentou com o aumento da dose de $\mathrm{K}_{2} \mathrm{O}$ quando se aplicaram $200 \mathrm{e} / \mathrm{ou} 100 \mathrm{~kg} \mathrm{ha}^{-1} \mathrm{de} \mathrm{N}$. Esse efeito foi observado em ambos os pomares, especialmente após armazenagem sob AC. Por outro lado, o teor de $\mathrm{SS}$ foi reduzido pela aplicação de altas doses de $\mathrm{N}$ no pomar sobre Cambissolo apenas quando a dose de $\mathrm{K}_{2} \mathrm{O}$ foi zero. No pomar sobre Neossolo, não houve efeitos significativos e/ou consistentes de doses de $\mathrm{N}$ sobre o teor de SS.

Redução do teor de SS devido ao aumento do teor de $\mathrm{N}$ tem sido relacionada ao efeito de diluição, devido ao aumento do tamanho dos frutos e/ou aumento da competição entre frutos e brotações por fotoassimilados (FERGUSON; BOYD, 2002). Por outro lado, o teor de SS em maçãs nem sempre aumenta pela adição de $\mathrm{K}_{2} \mathrm{O}$ (NEILSEN et al., 1998), embora o $\mathrm{K}$ tenha importante papel no aumento do transporte de açucares das folhas para os frutos (TAIZ; ZEIGER, 2006).
O presente estudo demonstra que a adubação nitrogenda e potássica pode alterar diferentemente o desenvolvimento e a qualidade de maçãs da cultivar Fuji, quando crescidas sobre Cambissolos e Neossolos de uma mesma região (São Joaquim$\mathrm{SC})$. A suplementação de $100 \mathrm{~kg} \mathrm{ha}^{-1}$ de $\mathrm{K}_{2} \mathrm{O}$ foi necessária no pomar sobre Cambissolo para que os frutos atingissem tamanhos e coloração vermelha para atender aos padrões mínimos de qualidade exigidos pelos mercados. No entanto, os benefícios da adição de $\mathrm{N}$ sobre o tamanho, ou do $\mathrm{K}_{2} \mathrm{O}$ sobre a coloração dos frutos no pomar sobre Neossolo, em detrimento da redução da firmeza da polpa, poderiam justificar- se para frutos destinados à exportação imediata ou à armazenagem por curtos períodos, mas não para frutos destinados à armazenagem por longos períodos. Isso sugere que as quantidades de $\mathrm{Ne} \mathrm{K}_{2} \mathrm{O}$ a serem suplementadas devem ser previstas com relativa precisão, para evitar perdas de produção, por reduzido tamanho de frutos e de qualidade, por baixa coloração associada a deficiências desses nutrientes, ou baixo potencial de armazenagem associado ao excesso desses nutrientes.

Os resultados do presente estudo também confirmam aqueles de Nava et al. (2008), mostrando que as respostas de macieiras à adubação nitrogenada e potássica, quando crescidas sobre solos pouco profundos e pedregosos de regiões Serranas, diferem muito daquelas crescidas sobre Latossolos profundos de regiões de Planalto (BASSO; SUZUKI, 1992; ERNANI; DIAS, 1999; ERNANI et al., 2000; ERNANI, 2008) no Sul do Brasil. Isso evidencia as diferentes capacidades desses solos em suprir nutrientes às macieiras.

TABELA 1- Atributos físico-químicos da camada arável dos solos $(0-20 \mathrm{~cm})$ nos pomares de maça plantados sobre Cambissolo e Neossolo. Análises feitas em 2004, nas parcelas que não receberam tratamentos com doses de $\mathrm{N} \mathrm{e} \mathrm{K}_{2} \mathrm{O}$ a partir de 1998 (ano de início do experimento).

\begin{tabular}{lcc}
\hline \multirow{2}{*}{ Atributo } & \multicolumn{2}{c}{ Tipo de solo } \\
\cline { 2 - 3 } & Cambissolo & Neossolo \\
\hline $\mathrm{pH}($ água $)$ & 6,6 & 6,7 \\
$\mathrm{P}\left(\mathrm{mg} \mathrm{dm}^{-3}\right)$ & 28 & 56 \\
$\mathrm{~K}\left(\mathrm{mg} \mathrm{dm}^{-3}\right)$ & 38 & 188 \\
$\mathrm{Ca}\left(\mathrm{mmol}_{\mathrm{c}} \mathrm{dm}^{-3}\right)$ & 109 & 95 \\
$\mathrm{Mg}\left(\mathrm{mmol}_{\mathrm{c}} \mathrm{dm}^{-3}\right)$ & 56 & 43 \\
Matéria orgânica $\left(\mathrm{g} \mathrm{dm}^{-3}\right)$ & 52 & 55 \\
Argila $\left(\mathrm{g} \mathrm{dm}^{-3}\right)$ & 300 & 300 \\
\hline
\end{tabular}


TABELA 2 - Teores minerais ( $\mathrm{mg} \mathrm{kg}^{-1}$ de massa fresca) em maçãs 'Fuji', provenientes de pomares plantados sobre Cambissolo e Neossolo, em resposta a doses $\left(\mathrm{kg} \mathrm{ha}^{-1}\right)$ de $\mathrm{N} \mathrm{e} \mathrm{K}_{2} \mathrm{O}$. São Joaquim- SC, dados médios dos anos de 2005 e 2006.

\begin{tabular}{|c|c|c|c|c|c|c|c|c|c|c|c|c|}
\hline \multirow{3}{*}{ Dose de N } & \multicolumn{4}{|c|}{ Cambissolo } & \multicolumn{8}{|c|}{ Neossolo } \\
\hline & \multicolumn{4}{|c|}{ Dose de $\mathrm{K}_{2} \mathrm{O}$} & \multirow[b]{2}{*}{$\mathbf{L}$} & \multirow[b]{2}{*}{$\mathbf{Q}$} & \multicolumn{4}{|c|}{ Dose de $\mathrm{K}_{2} \mathrm{O}$} & \multirow[b]{2}{*}{$\mathbf{L}$} & \multirow[b]{2}{*}{$\mathbf{Q}$} \\
\hline & $\mathbf{0}$ & 50 & 100 & 200 & & & $\mathbf{0}$ & 50 & 100 & 200 & & \\
\hline & \multicolumn{12}{|c|}{ Nitrogênio } \\
\hline $\mathbf{0}$ & 290 & 263 & 254 & 272 & $\mathrm{~ns}$ & $*$ & 262 & 271 & 251 & 255 & ns & ns \\
\hline 50 & 339 & 315 & 301 & 313 & $\mathrm{~ns}$ & ns & 257 & 271 & 277 & 303 & $*$ & ns \\
\hline 100 & 412 & 390 & 349 & 343 & $* * *$ & ns & 315 & 313 & 357 & 303 & ns & ns \\
\hline 200 & 438 & 465 & 361 & 315 & $* * *$ & $*$ & 342 & 364 & 330 & 321 & ns & ns \\
\hline $\mathbf{L}$ & $* * *$ & $* * *$ & $* * *$ & $*$ & & & $* * *$ & $* * *$ & $*$ & * & & \\
\hline $\mathbf{Q}$ & ns & ns & ns & $*$ & & & $\begin{array}{l}\mathrm{ns} \\
\text { sio }\end{array}$ & $*$ & ns & ns & & \\
\hline $\mathbf{0}$ & 784 & 1109 & 1205 & 1326 & $* * *$ & ns & 1349 & 1408 & 1439 & 1429 & ns & ns \\
\hline 50 & 797 & 832 & 1084 & 1331 & $* * *$ & ns & 1299 & 1357 & 1473 & 1430 & ns & ns \\
\hline 100 & 528 & 869 & 1118 & 1248 & $* * *$ & ns & 1374 & 1360 & 1389 & 1427 & ns & ns \\
\hline 200 & 506 & 698 & 1097 & 1227 & $* * *$ & ns & 1207 & 1317 & 1313 & 1407 & ns & ns \\
\hline $\mathbf{L}$ & $* * *$ & $* * *$ & ns & ns & & & ns & ns & ns & ns & & \\
\hline \multirow[t]{2}{*}{$\mathbf{Q}$} & ns & $\mathrm{ns}$ & ns & ns & & & ns & ns & ns & ns & & \\
\hline & \multicolumn{12}{|c|}{ Fósforo } \\
\hline $\mathbf{0}$ & 134 & 134 & 124 & 120 & $*$ & ns & 142 & 139 & 143 & 138 & ns & ns \\
\hline 50 & 136 & 121 & 125 & 127 & ns & ns & 141 & 124 & 134 & 144 & ns & $*$ \\
\hline 100 & 116 & 127 & 110 & 113 & ns & ns & 155 & 142 & 136 & 136 & ns & ns \\
\hline 200 & 108 & 119 & 110 & 107 & ns & ns & 122 & 122 & 124 & 143 & ns & ns \\
\hline $\mathbf{L}$ & $* *$ & ns & $*$ & $* *$ & & & $\mathrm{~ns}$ & $\mathrm{~ns}$ & ns & ns & & \\
\hline \multirow[t]{2}{*}{$\mathbf{Q}$} & ns & ns & ns & ns & & & $*$ & ns & ns & ns & & \\
\hline & \multicolumn{12}{|c|}{ Cálcio } \\
\hline $\mathbf{0}$ & 46 & 40 & 43 & 36 & $*$ & ns & 38 & 39 & 42 & 40 & ns & ns \\
\hline 50 & 49 & 41 & 34 & 37 & $* *$ & $*$ & 37 & 32 & 33 & 37 & ns & ns \\
\hline 100 & 40 & 33 & 32 & 31 & $* *$ & ns & 34 & 36 & 34 & 31 & ns & ns \\
\hline 200 & 42 & 37 & 37 & 37 & ns & ns & 36 & 36 & 36 & 41 & ns & ns \\
\hline $\mathbf{L}$ & ns & ns & $\mathrm{ns}$ & $\mathrm{ns}$ & & & ns & ns & ns & $\mathrm{ns}$ & & \\
\hline \multirow[t]{2}{*}{$\mathbf{Q}$} & ns & ns & $* *$ & $\mathrm{~ns}$ & & & ns & ns & ns & ns & & \\
\hline & \multicolumn{12}{|c|}{ Magnésio } \\
\hline $\mathbf{0}$ & 47 & 47 & 50 & 49 & ns & ns & 49 & 54 & 51 & 51 & ns & ns \\
\hline 50 & 48 & 48 & 48 & 48 & ns & ns & 48 & 48 & 49 & 50 & ns & ns \\
\hline 100 & 41 & 43 & 48 & 49 & ns & ns & 50 & 49 & 50 & 46 & ns & ns \\
\hline 200 & 35 & 43 & 45 & 49 & $*$ & ns & 47 & 51 & 50 & 50 & ns & ns \\
\hline $\mathbf{L}$ & $*$ & $\mathrm{~ns}$ & $\mathrm{~ns}$ & $\mathrm{~ns}$ & & & $\mathrm{~ns}$ & $\mathrm{~ns}$ & ns & ns & & \\
\hline $\mathbf{Q}$ & ns & ns & ns & ns & & & ns & ns & ns & ns & & \\
\hline
\end{tabular}

Efeito linear $(\mathrm{L})$ e quadrático $(\mathrm{Q})$ de doses de $\mathrm{N}_{\text {e }} \mathrm{K}_{2} \mathrm{O}$ analisado através de contrastes ortogonais polinomiais (ns, não significativo; $*$,** e $\mathrm{e}^{* * *}$, significativo a $5 ; 1$ e $0,1 \%$ de probabilidade de erro, respectivamente). 
TABELA 3 - Massa fresca, índice de iodo-amido e percentagem de cor vermelha de maçãs 'Fuji', analisadas um dia após a colheita, provenientes de pomares plantados sobre Cambissolo e Neossolo, em resposta a doses $\left(\mathrm{kg} \mathrm{ha}^{-1}\right)$ de $\mathrm{Ne} \mathrm{K}_{2} \mathrm{O}$. São Joaquim- SC, dados médios dos anos de 2005 e 2006.

\begin{tabular}{|c|c|c|c|c|c|c|c|c|c|c|c|c|}
\hline \multirow{4}{*}{ Dose de N } & \multicolumn{4}{|c|}{ Cambissolo } & \multicolumn{8}{|c|}{ Neossolo } \\
\hline & \multicolumn{4}{|c|}{ Dose de $\mathrm{K}_{2} \mathrm{O}$} & \multirow[b]{2}{*}{$\mathbf{L}$} & \multirow[b]{2}{*}{$\mathbf{Q}$} & \multicolumn{4}{|c|}{ Dose de K } & \multirow[b]{2}{*}{$\mathbf{L}$} & \multirow[b]{2}{*}{$\mathbf{Q}$} \\
\hline & 0 & $\mathbf{5 0}$ & 100 & 200 & & & $\mathbf{0}$ & 50 & 100 & 200 & & \\
\hline & \multicolumn{12}{|c|}{ Massa Fresca (g) } \\
\hline $\mathbf{0}$ & 127 & 147 & 148 & 161 & $* *$ & ns & 139 & 145 & 152 & 142 & ns & ns \\
\hline 50 & 124 & 146 & 169 & 156 & $*$ & $*$ & 145 & 162 & 143 & 172 & ns & ns \\
\hline 100 & 90 & 141 & 158 & 180 & $* * *$ & ns & 164 & 181 & 177 & 178 & ns & ns \\
\hline 200 & 76 & 126 & 159 & 161 & $* *$ & $*$ & 197 & 193 & 182 & 194 & ns & ns \\
\hline $\mathbf{L}$ & $* * *$ & $*$ & ns & ns & & & $* *$ & $* * *$ & $*$ & $* * *$ & & \\
\hline $\mathbf{Q}$ & $*$ & ns & ns & ns & & & ns & ns & $*$ & ns & & \\
\hline & \multicolumn{12}{|c|}{ Índice de Iodo Amido (1-9) } \\
\hline $\mathbf{0}$ & 4,9 & 3,8 & 4,0 & 4,0 & $*$ & $*$ & 3,6 & 3,5 & 3,7 & 3,4 & ns & ns \\
\hline 50 & 5,4 & 4,9 & 4,5 & 4,5 & $*$ & ns & 4,2 & 3,9 & 3,2 & 3,3 & $*$ & ns \\
\hline 100 & 6,7 & 5,7 & 5,1 & 4,6 & $* * *$ & $*$ & 3,8 & 4,1 & 4,2 & 3,8 & ns & ns \\
\hline 200 & 7,4 & 5,1 & 4,8 & 4,8 & $* * *$ & $* * *$ & 4,9 & 4,4 & 3,7 & 4,3 & ns & $* *$ \\
\hline $\mathbf{L}$ & $* * *$ & $* * *$ & ns & $* *$ & & & $* * *$ & $*$ & ns & $*$ & & \\
\hline $\mathbf{Q}$ & ns & $* * *$ & $* * *$ & ns & & & ns & ns & ns & $\mathrm{ns}$ & & \\
\hline & \multicolumn{12}{|c|}{ Cor Vermelha $(\%)$} \\
\hline 0 & 58 & 68 & 71 & 70 & $* * *$ & $* *$ & 78 & 80 & 80 & 84 & * & ns \\
\hline 50 & 58 & 65 & 66 & 68 & $* *$ & ns & 77 & 75 & 76 & 76 & ns & ns \\
\hline 100 & 51 & 56 & 60 & 63 & $* * *$ & ns & 64 & 77 & 59 & 79 & $* *$ & $*$ \\
\hline 200 & 46 & 54 & 60 & 61 & $* * *$ & $*$ & 66 & 64 & 61 & 76 & ns & $* * *$ \\
\hline $\mathbf{L}$ & $* * *$ & $* * *$ & $* * *$ & $* *$ & & & $*$ & $* * *$ & $* * *$ & $*$ & & \\
\hline $\mathbf{Q}$ & ns & ns & $*$ & ns & & & $* * *$ & ns & $* *$ & ns & & \\
\hline
\end{tabular}

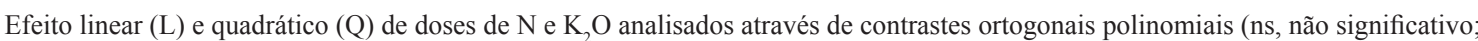
$*, * * \mathrm{e}^{* * *}$, significativo a $5 ; 1$ e $0,1 \%$ de probabilidade de erro, respectivamente).

TABELA 4- Firmeza da polpa (lb) de maçãs 'Fuji', analisadas um dia após a colheita e sete dias após armazenagem sob atmosfera do ar (AA) e atmosfera controlada (AC), provenientes de pomares plantados sobre Cambissolo e Neossolo, em resposta a doses $\left(\mathrm{kg} \mathrm{ha}^{-1}\right)$ de $\mathrm{N}$ e $\mathrm{K}_{2} \mathrm{O}$. São Joaquim-SC, s médios dos anos de 2005 e 2006.

\begin{tabular}{|c|c|c|c|c|c|c|c|c|c|c|c|c|}
\hline \multirow{3}{*}{ Dose de $\mathbf{N}$} & \multicolumn{4}{|c|}{ Cambissolo } & \multicolumn{8}{|c|}{ Neossolo } \\
\hline & \multicolumn{4}{|c|}{ Dose de $\mathrm{K}_{2} \mathrm{O}$} & \multirow[b]{2}{*}{$\mathbf{L}$} & \multirow[b]{2}{*}{$\mathbf{Q}$} & \multicolumn{4}{|c|}{ Dose de $\mathrm{K}_{2} \mathrm{O}$} & \multirow[b]{2}{*}{$\mathbf{L}$} & \multirow[b]{2}{*}{$\mathbf{Q}$} \\
\hline & $\mathbf{0}$ & 50 & 100 & 200 & & & $\mathbf{0}$ & 50 & 100 & 200 & & \\
\hline & \multicolumn{12}{|c|}{ Na Colheita } \\
\hline $\mathbf{0}$ & 18,6 & 17,9 & 16,9 & 17,3 & * & ns & 17,9 & 17,7 & 17,2 & 17,2 & $*$ & $\mathrm{~ns}$ \\
\hline 50 & 17,7 & 18,1 & 16,9 & 17,2 & $*$ & * & 17,3 & 16,9 & 17,4 & 16,8 & ns & $\mathrm{ns}$ \\
\hline 100 & 18,4 & 17,9 & 17,2 & 16,8 & $* * *$ & ns & 16,7 & 17,1 & 16,6 & 16,9 & ns & ns \\
\hline 200 & 19,7 & 18,0 & 17,6 & 17,0 & $* * *$ & $* * *$ & 16,6 & 16,0 & 16,6 & 16,5 & ns & $\mathrm{ns}$ \\
\hline $\mathbf{L}$ & $* * *$ & ns & * & ns & & & $* * *$ & $* * *$ & $* * *$ & ns & & \\
\hline \multirow[t]{2}{*}{$\mathbf{Q}$} & ** & ns & * & ns & & & ns & ns & ns & ns & & \\
\hline & \multicolumn{12}{|c|}{ Armazenagem sob AA } \\
\hline $\mathbf{0}$ & 15,2 & 14,5 & 13,7 & 12,9 & $* * *$ & $\mathrm{~ns}$ & 13,3 & 13,9 & 13,4 & 13,7 & ns & $\mathrm{ns}$ \\
\hline 50 & 13,6 & 14,1 & 12,7 & 13,0 & $*$ & ns & 12,5 & 12,9 & 12,9 & 12,5 & ns & $\mathrm{ns}$ \\
\hline 100 & 14,2 & 12,7 & 12,7 & 12,5 & $* *$ & $* * *$ & 11,8 & 12,1 & 12,3 & 12,2 & ns & $\mathrm{ns}$ \\
\hline 200 & 14,4 & 13,2 & 13,3 & 12,3 & $* * *$ & $\mathrm{~ns}$ & 11,5 & 11,5 & 11,4 & 12,1 & ns & ns \\
\hline L & $*$ & $* * *$ & $*$ & $* *$ & & & $* * *$ & $* * *$ & $* * *$ & $* *$ & & \\
\hline \multirow[t]{2}{*}{$\mathbf{Q}$} & $* *$ & $* *$ & $* * *$ & ns & & & ns & ns & ns & $*$ & & \\
\hline & \multicolumn{12}{|c|}{ Armazenagem sob AC } \\
\hline 0 & 18,8 & 17,7 & 16,2 & 16,2 & $* * *$ & $* *$ & 16,7 & 17,4 & 17,0 & 17,2 & ns & $\mathrm{ns}$ \\
\hline 50 & 17,7 & 18,0 & 16,2 & 16,6 & $* *$ & $\mathrm{~ns}$ & 16,7 & 16,0 & 16,7 & 16,4 & ns & $\mathrm{ns}$ \\
\hline 100 & 18,2 & 17,2 & 16,2 & 15,6 & $* * *$ & $*$ & 15,9 & 15,9 & 16,2 & 16,0 & ns & ns \\
\hline 200 & 18,9 & 17,7 & 17,1 & 16,4 & $* * *$ & $*$ & 15,4 & 15,4 & 15,5 & 15,7 & ns & $\mathrm{ns}$ \\
\hline \multirow{2}{*}{$\begin{array}{l}\mathbf{L} \\
\mathrm{Q}\end{array}$} & \multirow{2}{*}{$\begin{array}{c}10, \\
\text { ns } \\
* * *\end{array}$} & $\mathrm{~ns}$ & \multirow{2}{*}{$\begin{array}{c}\mathrm{ns} \\
*\end{array}$} & $\mathrm{~ns}$ & & & $* * *$ & $* *$ & $* *$ & $* * *$ & & \\
\hline & & ns & & ns & & & ns & $*$ & ns & $\mathrm{ns}$ & & \\
\hline
\end{tabular}

Efeito linear (L) e quadrático $(\mathrm{Q})$ de doses de $\mathrm{N} \mathrm{e} \mathrm{K}_{2} \mathrm{O}$ analisados através de contrastes ortogonais polinomiais (ns, não significativo; $*, * * \mathrm{e}^{* * *}$, significativo a $5 ; 1$ e $0,1 \%$ de probabilidade de erro, respectivamente). 
TABELA 5 - Acidez titulável (\%) de maçãs 'Fuji', analisadas um dia após a colheita e sete dias após a armazenagem sob atmosfera do ar (AA) e atmosfera controlada (AC), provenientes de pomares plantados sobre Cambissolo e Neossolo, em resposta a doses $\left(\mathrm{kg} \mathrm{ha}^{-1}\right)$ de $\mathrm{N}$ e $\mathrm{K}_{2} \mathrm{O}$. São Joaquim-SC, dados médios dos anos de 2005 e 2006.

\begin{tabular}{|c|c|c|c|c|c|c|c|c|c|c|c|c|}
\hline \multirow{3}{*}{ Dose de $N$} & \multicolumn{4}{|c|}{ Cambissolo Neossolo } & & & \multicolumn{4}{|c|}{ Neossolo } & \multirow[b]{3}{*}{$\mathbf{L}$} & \multirow[b]{3}{*}{$\mathbf{Q}$} \\
\hline & \multicolumn{4}{|c|}{ Dose de $\mathrm{K}_{2} \mathrm{O}$} & \multirow[b]{2}{*}{$\mathbf{L}$} & \multirow[b]{2}{*}{$\mathbf{Q}$} & \multicolumn{4}{|c|}{ Dose de $\mathrm{K}_{2} \mathrm{O}$} & & \\
\hline & $\mathbf{0}$ & 50 & 100 & 200 & & & $\mathbf{0}$ & $\mathbf{5 0}$ & 100 & 200 & & \\
\hline & \multicolumn{12}{|c|}{ Na Colheita } \\
\hline $\mathbf{0}$ & 0,27 & 0,335 & 0,348 & 0,411 & $* * *$ & $\mathrm{~ns}$ & 0,452 & 0,439 & 0,474 & 0,424 & $\mathrm{~ns}$ & $\mathrm{~ns}$ \\
\hline 50 & 0,25 & 0,251 & 0,312 & 0,403 & $* * *$ & ns & 0,399 & 0,410 & 0,433 & 0,455 & ns & $\mathrm{ns}$ \\
\hline 100 & 0,16 & 0,263 & 0,331 & 0,382 & $* * *$ & $* *$ & 0,378 & 0,423 & 0,399 & 0,412 & ns & $\mathrm{ns}$ \\
\hline 200 & 0,16 & 0,227 & 0,298 & 0,366 & $* * *$ & ns & 0,370 & 0,386 & 0,413 & 0,470 & **** & ns \\
\hline $\mathbf{L}$ & $* * *$ & $* *$ & ns & $*$ & & & * & * & ns & ns & & \\
\hline $\mathbf{Q}$ & $\mathrm{ns}$ & ns & ns & ns & & & ns & ns & ns & ns & & \\
\hline \multicolumn{13}{|c|}{ Armazenagem sob AA } \\
\hline $\mathbf{0}$ & 0,08 & 0,131 & 0,140 & 0,161 & $* * *$ & $\mathrm{~ns}$ & 0,192 & 0,188 & 0,183 & 0,194 & ns & $\mathrm{ns}$ \\
\hline 50 & 0,08 & 0,084 & 0,124 & 0,152 & **** & $\mathrm{ns}$ & 0,179 & 0,182 & 0,174 & 0,191 & ns & $\mathrm{ns}$ \\
\hline 100 & 0,05 & 0,079 & 0,115 & 0,142 & $* * *$ & $\mathrm{~ns}$ & 0,162 & 0,181 & 0,173 & 0,175 & $\mathrm{~ns}$ & $\mathrm{~ns}$ \\
\hline 200 & 0,05 & 0,068 & 0,094 & 0,130 & $* * *$ & ns & 0,133 & 0,165 & 0,168 & 0,166 & $* *$ & $\mathrm{~ns}$ \\
\hline $\mathbf{L}$ & $* *$ & $* * *$ & $* *$ & $*$ & & & $* * *$ & ns & $\mathrm{ns}$ & $* *$ & & \\
\hline $\mathbf{Q}$ & $\mathrm{ns}$ & * & ns & ns & & & ns & ns & ns & ns & & \\
\hline \multicolumn{13}{|c|}{ Armazenagem sob AC } \\
\hline $\mathbf{0}$ & 0,17 & 0,210 & 0,271 & 0,312 & $* * *$ & ns & 0,325 & 0,336 & 0,333 & 0,318 & $\mathrm{~ns}$ & $\mathrm{~ns}$ \\
\hline 50 & 0,14 & 0,163 & 0,232 & 0,299 & $* * *$ & ns & 0,303 & 0,324 & 0,318 & 0,333 & $*$ & ns \\
\hline 100 & 0,10 & 0,162 & 0,231 & 0,263 & $* * *$ & ns & 0,284 & 0,296 & 0,316 & 0,315 & * & ns \\
\hline 200 & 0,09 & 0,146 & 0,217 & 0,269 & $* * *$ & ns & 0,243 & 0,301 & 0,282 & 0,338 & $* * *$ & ns \\
\hline $\mathbf{L}$ & $* * *$ & $*$ & ns & ns & & & $* * *$ & $* *$ & $* *$ & ns & & \\
\hline Q & $\mathrm{ns}$ & ns & ns & ns & & & ns & ns & ns & ns & & \\
\hline
\end{tabular}

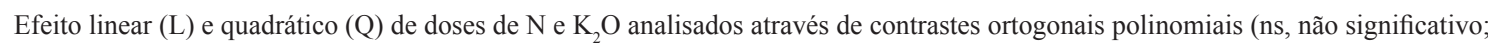
*, ** e $* * *$, significativo a $5 ; 1$ e $0,1 \%$ de probabilidade de erro, respectivamente).

TABELA 6 - Teores de sólidos solúveis (\%) de maçãs 'Fuji', analisadas um dia após a colheita e sete dias após a armazenagem sob atmosfera do ar (AA) e atmosfera controlada (AC), provenientes de pomares plantados sobre Cambissolo e Neossolo, em resposta a doses de $\mathrm{N}_{\text {e }} \mathrm{K}_{2} \mathrm{O}$. São Joaquim-SC, dados médios dos anos de 2005 e 2006.

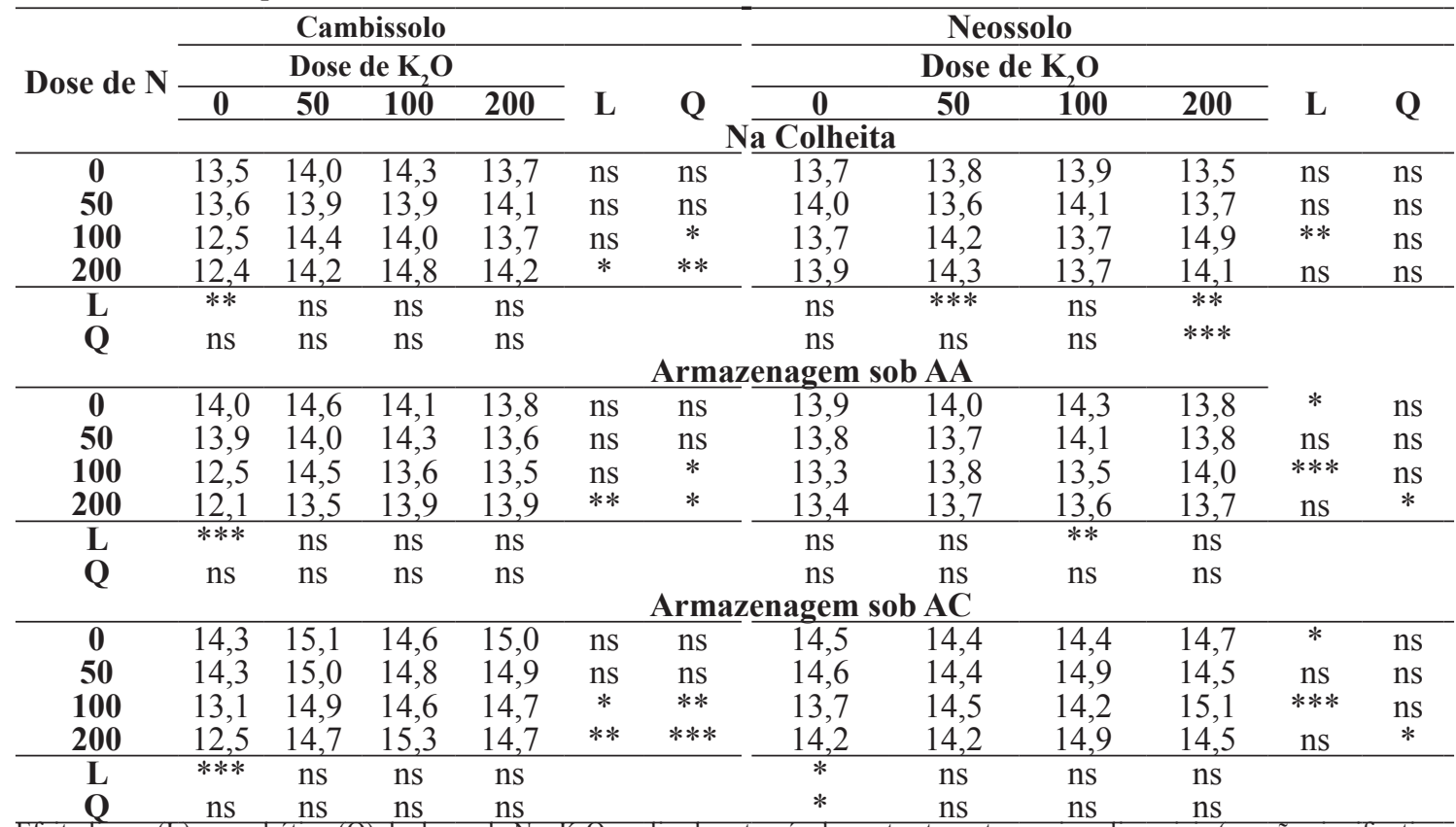




\section{CONCLUSÕES}

1-Aplicações de altas doses de $\mathrm{N}$ aumentam o teor de $\mathrm{N}$ e diminuem o teor de amido e a cor vermelha nos frutos, em ambos os solos e independentemente da dose de $\mathrm{K}_{2} \mathrm{O}$.

2-A massa dos frutos aumenta e a firmeza da polpa diminui em resposta a altas doses de $\mathrm{N}$ aplicadas no Neossolo, independentemente da dose de $\mathrm{K}_{2} \mathrm{O}$, mas não são consistentemente afetadas quando $\mathrm{N}$ é aplicado sobre o Cambissolo.

3-A acidez titulável (AT) dos frutos é diminuída pela aplicação de altas doses de $\mathrm{N}$ em ambos os solos, quando se aplicam baixas doses de $\mathrm{K}_{2} \mathrm{O}$.

4-Aplicações de altas doses de $\mathrm{K}_{2} \mathrm{O}$ aumentam o teor de $\mathrm{K}$ e a massa dos frutos crescidos sobre Cambissolo, mas não naqueles crescidos sobre $\mathrm{Ne}$ ossolo, independentemente da dose de N.

5-O teor de amido, a AT e a coloração avermelhada aumentam, enquanto a firmeza diminui em resposta a altas doses de $\mathrm{K}_{2} \mathrm{O}$ no Cambissolo, independentemente da dose de $\mathrm{N}$, mas não são consistentemente afetadas em resposta a altas doses de $\mathrm{K}_{2} \mathrm{O}$ no Neossolo.

\section{AGRADECIMENTOS}

À Associação Brasileira de Produtores de Maçãs (ABPM) e a Fundação de Amparo à Pesquisa e Inovação do Estado de Santa Catarina (FAPESC), pelo apoio financeiro, a Miguel Ângelo de Rocco, Marcelo J. Vieira e Andréia M. T. Scolaro, pelo apoio na execução dos experimentos, e à CAPES, pela Bolsa de Mestrado ao primeiro autor.

\section{REFERÊNCIAS}

ARGENTA, L.C.; VIEIRA, M.J.; SCOLARO, A.M.T. Validação de catálogos de cores como indicadores do estádio de maturação e ponto de colheita de maçã. Agropecuária Catarinense, Florianópolis, v.32, n.3, p.71-77, 2010.

BASSO, C.; SUZUKI, A. Resposta da macieira cv. Golden Delicious à adubação nitrogenada. Revista Brasileira de Ciência do Solo, Campinas, v. 16, n.2, p.223-227, 1992.

CQFS - COMISSÃO DE QUÍMICA E FERTILIDADE DO SOLO. Manual de adubação e calagem para os estados do Rio Grande do Sul e de Santa Catarina. 10. ed. Porto Alegre, 2004. 400 p.
DRAKE, S.R.; RAESE, J.T.; SMITH, T.J. Time of nitrogen application and its influence on 'Golden Delicious' apple yield and fruit quality. Journal of Plant Nutrition, Philadelphia, v.25, n.1, p.143-157, 2002.

ERNANI, P.R.; DIAS, J. Soil nitrogen application in the spring did not increase apple yield. Ciência Rural, Santa Maria, v.29, n.4, p.645-649, 1999.

ERNANI P.R.; DIAS, J.; BORGES, M. A aplicação de nitrogênio ao solo em diferentes estádios não afetou o rendimento de frutos de cultivares de macieira. Ciência Rural, Santa Maria, v.30, n.2, p.223-227, 2000.

ERNANI, P.R.; DIAS, J.; FLORE, J.A. Annual additions of potassium to the soil increased apple yield in Brazil. Communications in Soil Science and Plant Analysis, New York, v.33, n.7-8, p.12911304, 2002

FALLAHI, E. Productivity, postharvest physiology, and soil nitrate movement as influenced by nitrogen applications to 'Delicious' apple. Acta Horticulturae, Wageningen, n.512, p.149-157, 2000.

FALLAHI, E.; COLT, W.M.; BAIRD, C.R.; FALLAHI, B.; CHUN, I.J. Influence of nitrogen and bagging on fruit quality and mineral concentrations of 'Fuji' apple. HortTechnology, Alexandria, v.11, n.3, p.462-466, 2001.

FALLAHI, E.; FALLAHI, B.; NEILSEN, G.H.; NEILSEN, D.; PERYEA, F.J. Effects of mineral nutrition on fruit quality and nutritional disorders in apples. Acta Horticulturae, Wageningen, n.868, p.49-60, 2010

FAUST, M. Nutrition of fruit trees. In: FAUST, M. Physiology of temperate zone fruit trees. New York: Wiley \& Sons, 1989. p.53-132.

FERGUSON, I.B.; BOYD, L.M. Inorganic nutrients and fruit quality. In: KNEE, M. Fruit quality and its biological basis. Ohio: Wiley Blackwell, 2002. p.17-45.

HUNSCHE, M.; BRACKMANN, A.; ERNANI, P.R. Efeito da adubação potássica na qualidade póscolheita de maçãs 'Fuji'. Pesquisa Agropecuária Brasileira, Brasília, v.38, n.4, p.489-496, 2003. 
JOHNSON, R.S. Manipulating vegetative and reproductive growth with water and nitrogen. In: MAIB, K. Tree fruit physiology: growth \& development. Washington: Good Fruit Grower, 1996, p.81-87.

LITTLE, C.R.; HOLMES, R.J. Preharvest factors affecting storage. In: LITTLE, C.R.; HOLMES, R.J. Storage technology for apples and pears. Victoria: Department of Natural Resources and Environment, 2000. p. 54-110.

NAVA, G.; DECHEN, A.R. Long-term annual fertilization with nitrogen and potassium affect yield and mineral composition of 'Fuji' apple. Scientia Agricola, Piracicaba, v.66, n.3, p.377-385, 2009.

NAVA, G.; DECHEN, A.R.; NACHTIGALL, G.R. Nitrogen and potassium fertilization affect apple fruit quality in Southern Brazil. Communications in Soil Science and Plant Analysis, New York, v.39, n. 1/2, p.96-107, 2008.

NEILSEN, D.; NEILSEN, G.H.; HALL, J.W. Fruit mineral concentration and quality of 'Gala' apples as affected by rate and timing of fertigated N. Acta Horticulturae, Wageningen, n.512, p.159-167, 2000.

NEILSEN, G.; PARCHOMCHUK, P.; MEHERIUK, M.; NEILSEN, D. Development and correction of K-deficiency in drip-irrigated apple. HortScience, Alexandria, v.33, n.2, p.258-261, 1998.
NEILSEN, G.H.; NEILSEN, D. The effect of $\mathrm{K}$-fertilization on apple fruit $\mathrm{Ca}$ concentration and quality. Acta Horticulturae, Wageningen, n.721, p.177-183, 2006.

RAESE, J.T.; DRAKE S.R. Nitrogen fertilization and elemental composition affects fruit quality of 'Fuji' apples. Journal of Plant Nutrition, Philadelphia, v.20, n.12, p.1797-1809, 1997.

RAESE, J.T.; DRAKE S.R.; CURRY, E.A. Nitrogen fertilizer influences fruit quality, soil nutrients and cover crops, leaf color and nitrogen content, biennial bearing and cold hardiness of 'Golden Delicious'. Journal of Plant Nutrition, Philadelphia, v.30, n.10, p.1585-1604, 2007.

TAIZ, L.; ZEIGER, E. Plant physiology. 4. ed. Sunderland: Sinauer Associates, 2006. 705p.

TEDESCO, M.J.; GIANELLO, C.; BISSANI, C.A.; BOHNEN, H.; VOLKWEISS, S.J. Análise do solo, plantas e outros materiais. 2.ed. Porto Alegre: Departamento de Solos, UFRGS, 1995. 174p. (Boletim Técnico de Solos, 5).

WARGO, J.M.; MERWIN, I.A.; WATKINS, C.B. Fruit size, yield, and market value of 'GoldRush' apple are affected by amount, timing and method of nitrogen fertilization. HortTechnology, Alexandria, v.13, p.153-161, 2003. 